\title{
ALTERNATING DOUBLE EULER SUMS, HYPERGEOMETRIC IDENTITIES AND A THEOREM OF ZAGIER
}

\author{
LEE-PENG TEO
}

\begin{abstract}
In this work, we derive relations between generating functions of double stuffle relations and double shuffle relations to express the alternating double Euler sums $\zeta(\bar{r}, s), \zeta(r, \bar{s})$ and $\zeta(\bar{r}, \bar{s})$ with $r+s$ odd in terms of zeta values. We also give a direct proof of a hypergeometric identity which is a limiting case of a basic hypergeometric identity of Andrews. Finally, we gave another proof for the formula of Zagier on the multiple zeta values $\zeta(2, \ldots, 2,3,2, \ldots, 2)$.
\end{abstract}

\section{Introduction}

Following [15], for positive integers $k_{1}, \ldots, k_{n}$ with $k_{n} \geq 2$, define the multiple zeta values and multiple zeta star values by

$$
\begin{aligned}
\zeta\left(k_{1}, \ldots, k_{n}\right) & =\sum_{1 \leq m_{1}<\ldots<m_{n}} \frac{1}{m_{1}^{k_{1}} \ldots m_{n}^{k_{n}}}, \\
\zeta^{\star}\left(k_{1}, \ldots, k_{n}\right) & =\sum_{1 \leq m_{1} \leq \ldots \leq m_{n}} \frac{1}{m_{1}^{k_{1}} \ldots m_{n}^{k_{n}}} .
\end{aligned}
$$

When $n=2$, the double sum

$$
\zeta(r, s)=\sum_{m=2}^{\infty} \frac{1}{m^{s}} \sum_{j=1}^{k-1} \frac{1}{j^{r}}
$$

has been considered by Euler. Hence, multiple zeta values are also known as Euler sums.

Date: September 8, 2021.

2000 Mathematics Subject Classification. Primary 33E20, 33C20.

Key words and phrases. Double Euler sums, Hypergeometric identities, Multiple zeta values, Multiple zeta star values .

This work was started when I visited J. H. Teh in National Tsing Hua University of Taiwan at the end of 2014. I would like to thank NTHU for their hospitality and J. H. Teh for the helpful discussions. 
As in [3], we can also define the following alternating double Euler sums

$$
\begin{aligned}
\zeta(\bar{r}, s) & =\sum_{m=2}^{\infty} \frac{1}{m^{s}} \sum_{j=1}^{m-1} \frac{(-1)^{j}}{j^{r}}, \\
\zeta(r, \bar{s}) & =\sum_{m=2}^{\infty} \frac{(-1)^{m}}{m^{s}} \sum_{j=1}^{m-1} \frac{1}{j^{r}}, \\
\zeta(\bar{r}, \bar{s}) & =\sum_{m=2}^{\infty} \frac{(-1)^{m}}{m^{s}} \sum_{j=1}^{m-1} \frac{(-1)^{j}}{j^{r}} .
\end{aligned}
$$

These can also be considered as extensions of the alternating series

$$
\zeta(\bar{k})=\sum_{m=1}^{\infty} \frac{(-1)^{m}}{m^{k}}
$$

to double Euler sums. Similar extensions can also be defined for multiple zeta values and multiple zeta star values. It is well-known that

$$
\zeta(\bar{k})=-\left(1-2^{1-k}\right) \zeta(k) .
$$

In [15], Zagier studied the multiple zeta value

$$
H(a, b)=\zeta(\underbrace{2, \ldots, 2}_{a}, 3, \underbrace{2, \ldots, 2}_{b})
$$

and the multiple zeta star value

$$
H^{\star}(a, b)=\zeta^{\star}(\underbrace{2, \ldots, 2}_{a}, 3, \underbrace{2, \ldots, 2}_{b}) .
$$

In particular, he proved the following formulas.

Theorem 1.1. Let

$$
\begin{gathered}
H(a)=\zeta(\underbrace{2, \ldots, 2}_{a}), \\
H^{\star}(a)=\zeta^{\star}(\underbrace{2, \ldots, 2}_{a}) .
\end{gathered}
$$

Then

$$
\begin{gathered}
H(a, b)=2 \sum_{r=1}^{K}(-1)^{r}\left\{\left(\begin{array}{c}
2 r \\
2 a+2
\end{array}\right) \zeta(2 r+1)+\left(\begin{array}{c}
2 r \\
2 b+1
\end{array}\right) \zeta \overline{(2 r+1)}\right\} H(K-r), \\
H^{\star}(a, b)=-2 \sum_{r=1}^{K}\left\{\left[\left(\begin{array}{c}
2 r \\
2 a
\end{array}\right)-\delta_{r, a}\right] \zeta(2 r+1)+\left(\begin{array}{c}
2 r \\
2 b+1
\end{array}\right) \zeta \overline{(2 r+1)}\right\} H^{\star}(K-r),
\end{gathered}
$$

where $K=a+b+1$.

The formula for $H(a, b)$ is needed in the proof of Hoffman conjecture by $\mathrm{F}$. Brown [4, which states that all multiple zeta values can be expressed as $\mathbb{Q}$ - linear combinations of the multiple zeta values $\zeta\left(k_{1}, \ldots, k_{n}\right)$ with each $k_{i}$ equals to 2 or 3. 
The proof provided by Zagier for Theorem 1.1] uses complex analytic methods to show that the generating functions of both sides of the formula are equal. In [12], Li provided an alternative proof using transformations of hypergeometric series ${ }_{3} F_{2}$. Recently, Pilehrood and Pilehrood [9] gave another proof using a special hypergeometric identity stated in 11, which can be considered as a limiting case of a basic hypergeometric identity of Andrews [2. Pilehrood and Pilehrood 9] proved the following result:

Theorem 1.2.

$$
H^{\star}(a, b)=-4 \zeta(2 a+1, \overline{2 b+2})-2 \zeta(\overline{2 a+2 b+3}) .
$$

As a matter of fact, this identity has been proved by Pilehrood, Pilehrood and Tauraso in [10]. From this identity, Pilehrood and Pilehrood [9] proved Theorem 1.1 using complex analytic method.

As was pointed out in [10, the formula for $H^{\star}(a, b)$ given in Theorem 1.1 is actually an immediate consequence of Theorem 1.2 and one of the formulas in the following theorem.

Theorem 1.3. If $k=r+s \geq 3$ is an odd positive integer, then

$$
\begin{aligned}
\zeta(r, s)= & -\frac{1}{2} \zeta(k)+\frac{1+(-1)^{s}}{2} \zeta(r) \zeta(s) \\
& +(-1)^{r} \sum_{l=0}^{\frac{k-1}{2}}\left[\left(\begin{array}{c}
k-2 l-1 \\
r-1
\end{array}\right) \zeta(k-2 l)+\left(\begin{array}{c}
k-2 l-1 \\
s-1
\end{array}\right) \zeta(k-2 l)\right] \zeta(2 l), \\
\zeta(\bar{r}, s)= & -\frac{1}{2} \zeta(\bar{k})+\frac{1+(-1)^{s}}{2} \zeta(\bar{r}) \zeta(s) \\
& +(-1)^{r} \sum_{l=0}^{\frac{k-1}{2}}\left[\left(\begin{array}{c}
k-2 l-1 \\
r-1
\end{array}\right) \zeta(\overline{k-2 l})+\left(\begin{array}{c}
k-2 l-1 \\
s-1
\end{array}\right) \zeta(k-2 l)\right] \zeta(\overline{2 l}), \\
\zeta(r, \bar{s})= & -\frac{1}{2} \zeta(\bar{k})+\frac{1+(-1)^{s}}{2} \zeta(r) \zeta(\bar{s}) \\
& +(-1)^{r} \sum_{l=0}^{\frac{k-1}{2}}\left[\left(\begin{array}{c}
k-2 l-1 \\
r-1
\end{array}\right) \zeta(k-2 l)+\left(\begin{array}{c}
k-2 l-1 \\
s-1
\end{array}\right) \zeta(\overline{k-2 l})\right] \zeta(\overline{2 l}), \\
\zeta(\bar{r}, \bar{s})= & -\frac{1}{2} \zeta(k)+\frac{1+(-1)^{s}}{2} \zeta(\bar{r}) \zeta(\bar{s}) \\
& +(-1)^{r} \sum_{l=0}^{\frac{k-1}{2}}\left[\left(\begin{array}{c}
k-2 l-1 \\
r-1
\end{array}\right) \zeta(\overline{k-2 l})+\left(\begin{array}{c}
k-2 l-1 \\
s-1
\end{array}\right) \zeta(\overline{k-2 l})\right] \zeta(2 l) .
\end{aligned}
$$

One observes some symmetries among these four formulas. The first formula (1.11) in this theorem was proved in [3] using matrices, and the other three formulas (1.12)-(1.14) were mentioned in the same paper but the details of proofs were not given. Proofs of these formulas were given in [7] using contour integral 
representations. The formula for $H^{\star}(a, b)$ given in Theorem 1.1 is an immediate consequence of Theorem 1.2 and the third formula in Theorem 1.3 .

In this work, we are going to prove the formulas (1.12)-(1.14) in Theorem 1.3 using the method used by Zagier 15 to prove the formula (1.11). This method has been outlined in 8 and explored in [5]. As pointed out in [13, the formulas in Theorem 1.3 can also be obtained by taking the $q \rightarrow 1$ limits of the corresponding $q$-analogs proved in [14. Nevertheless, we find it worthwhile to present the proof along the line of Zagier [15].

After proving the formulas (1.12)-(1.14), we give a direct proof to the hypergeometric identity used in 9 to prove Theorem [1.2, which is of interest in its own right. We then give a slightly simpler proof to Theorem 1.2 From this, the formula (1.9) for $H^{\star}(a, b)$ in Theorem 1.1 follows immediately. We then prove the formula (1.8) for $H(a, b)$ in Theorem 1.1 from (1.9), which better reflects the symmetries between these two formulas.

\section{Alternating Double Euler Sums}

In this section, we prove the formulas (1.12)-(1.14) in Theorem 1.3 using the method of Zagier [15, 8]. First we have the following well-known double-stuffle relations.

Lemma 2.1. For $r \geq 1, s \geq 2$,

$$
\zeta(\bar{r}) \zeta(s)=\zeta(\bar{r}, s)+\zeta(s, \bar{r})+\zeta(\overline{r+s}) .
$$

For $r \geq 1, s \geq 1$,

$$
\zeta(\bar{r}) \zeta(\bar{s})=\zeta(\bar{r}, \bar{s})+\zeta(\bar{s}, \bar{r})+\zeta(r+s) .
$$

Proof. We note that $\zeta(\overline{1})=\log 2$ is well-defined. Eq. (2.1) follows from

$$
\sum_{m=1}^{\infty} \frac{(-1)^{m}}{m^{r}} \sum_{j=1}^{\infty} \frac{1}{j^{s}}=\sum_{m=1}^{\infty} \frac{(-1)^{m}}{m^{r}} \sum_{j=1}^{m-1} \frac{1}{j^{s}}+\sum_{m=1}^{\infty} \frac{(-1)^{m}}{m^{r}} \frac{1}{m^{s}}+\sum_{m=1}^{\infty} \frac{(-1)^{m}}{m^{r}} \sum_{j=m+1}^{\infty} \frac{1}{j^{s}} .
$$

Eq. (2.2) is proved in the same way.

When $r \geq 1, \zeta(r, \overline{1})$ and $\zeta(\bar{r}, \overline{1})$ are convergent. However, $\zeta(\bar{r}, s)$ is convergent only if $s \geq 2$. As in [15, we define $\zeta(1)$ symbolically as $T$. For $r \geq 1$, let

$$
\zeta(\bar{r}, 1)=\zeta(\bar{r}) T-\zeta(1, \bar{r})-\zeta(\overline{r+1}) .
$$

Then the relation (2.1) still holds symbolically for $s=1$.

Fix an integer $k$, we define the following generating functions:

$$
\begin{aligned}
& F_{1}(x, y)=\sum_{\substack{r, s \geq 1 \\
r+s=k}} \zeta(\bar{r}) \zeta(s) x^{r-1} y^{s-1}, \\
& F_{2}(x, y)=\sum_{\substack{r, s \geq 1 \\
r+s=k}} \zeta(\bar{r}) \zeta(\bar{s}) x^{r-1} y^{s-1},
\end{aligned}
$$




$$
\begin{gathered}
G_{1}(x, y)=\sum_{\substack{r, s \geq 1 \\
r+s=k}} \zeta(\bar{r}, s) x^{r-1} y^{s-1}, \\
G_{2}(x, y)=\sum_{\substack{r, s \geq 1 \\
r+s=k}} \zeta(r, \bar{s}) x^{r-1} y^{s-1}, \\
G_{3}(x, y)=\sum_{\substack{r, s \geq 1 \\
r+s=k}} \zeta(\bar{r}, \bar{s}) x^{r-1} y^{s-1}, \\
T_{1}(x, y)=\zeta(k) \sum_{\substack{r, s \geq 1 \\
r+s=k}} x^{r-1} y^{s-1}=\zeta(k) \frac{x^{k-1}-y^{k-1}}{x-y}, \\
T_{2}(x, y)=\zeta(\bar{k}) \sum_{\substack{r, s \geq 1 \\
r+s=k}} x^{r-1} y^{s-1}=\zeta(\bar{k}) \frac{x^{k-1}-y^{k-1}}{x-y} .
\end{gathered}
$$

Notice that only $F_{2}(x, y), T_{1}(x, y)$ and $T_{2}(x, y)$ are symmetric in $x$ and $y$. The relations in Lemma 2.1 translate into the following identities:

\section{Theorem 2.2.}

$$
\begin{aligned}
& F_{1}(x, y)=G_{1}(x, y)+G_{2}(y, x)+T_{2}(x, y), \\
& F_{2}(x, y)=G_{3}(x, y)+G_{3}(y, x)+T_{1}(x, y) .
\end{aligned}
$$

Next, we derive the double-shuffle relation for alternating double Euler sums. Notice that

$$
\begin{aligned}
\zeta(r) & =\sum_{m=1}^{\infty} \frac{1}{m^{r}} \\
& =\frac{1}{\Gamma(r)} \int_{0}^{\infty} t^{r-1} \sum_{n=1}^{\infty} e^{-t n} d t \\
& =\frac{1}{\Gamma(r)} \int_{0}^{\infty} \frac{t^{r-1}}{e^{t}-1} d t .
\end{aligned}
$$

In the same way, one can derive the formula

$$
\zeta(\bar{r})=-\frac{1}{\Gamma(r)} \int_{0}^{\infty} \frac{t^{r-1}}{e^{t}+1} d t .
$$

For alternating double zeta values, we have

\section{Lemma 2.3.}

$$
\begin{aligned}
& \zeta(\bar{r}, s)=-\frac{1}{\Gamma(r) \Gamma(s)} \int_{0}^{\infty} \int_{0}^{\infty} \frac{t^{s-1} u^{r-1}}{\left(e^{t}-1\right)\left(e^{t+u}+1\right)} d u d t \\
& \zeta(r, \bar{s})=\frac{1}{\Gamma(r) \Gamma(s)} \int_{0}^{\infty} \int_{0}^{\infty} \frac{t^{s-1} u^{r-1}}{\left(e^{t}+1\right)\left(e^{t+u}+1\right)} d u d t \\
& \zeta(\bar{r}, \bar{s})=-\frac{1}{\Gamma(r) \Gamma(s)} \int_{0}^{\infty} \int_{0}^{\infty} \frac{t^{s-1} u^{r-1}}{\left(e^{t}+1\right)\left(e^{t+u}-1\right)} d u d t .
\end{aligned}
$$


Proof.

$$
\begin{aligned}
\zeta(\bar{r}, s) & =\sum_{m=1}^{\infty} \frac{1}{m^{s}} \sum_{j=1}^{m-1} \frac{(-1)^{j}}{j^{r}} \\
& =\sum_{j=1}^{\infty} \frac{(-1)^{j}}{j^{r}} \sum_{m=1}^{\infty} \frac{1}{(m+j)^{s}} \\
& =\frac{1}{\Gamma(r) \Gamma(s)} \int_{0}^{\infty} \int_{0}^{\infty} t^{s-1} u^{r-1} \sum_{j=1}^{\infty}(-1)^{j} \sum_{m=1}^{\infty} e^{-t(m+j)} e^{-u j} d u d t \\
& =-\frac{1}{\Gamma(r) \Gamma(s)} \int_{0}^{\infty} \int_{0}^{\infty} \frac{t^{s-1} u^{r-1}}{\left(e^{t}-1\right)\left(e^{t+u}+1\right)} d u d t .
\end{aligned}
$$

The other two formulas are proved in the same way.

For the usual double Euler sum, the shuffle relation reads as

$$
\zeta(r) \zeta(s)=\sum_{j=1}^{k-1}\left(\begin{array}{l}
j-1 \\
r-1
\end{array}\right) \zeta(k-j, j)+\sum_{j=1}^{k-1}\left(\begin{array}{l}
j-1 \\
s-1
\end{array}\right) \zeta(k-j, j)
$$

when $r \geq 1, s \geq 2$ and $r+s=k$. Using the integral representations given in Lemma 2.3 . we can prove the following shuffle relations for alternating double Euler sums:

Theorem 2.4. If $r \geq 1, s \geq 2, r+s=k$, then

$$
\zeta(\bar{r}) \zeta(s)=\sum_{j=1}^{k-1}\left(\begin{array}{l}
j-1 \\
r-1
\end{array}\right) \zeta(\overline{k-j}, \bar{j})+\sum_{j=1}^{k-1}\left(\begin{array}{l}
j-1 \\
s-1
\end{array}\right) \zeta(\overline{k-j}, j) .
$$

If $r, s \geq 1, r+s=k$, then

$$
\zeta(\bar{r}) \zeta(\bar{s})=\sum_{j=1}^{k-1}\left(\begin{array}{l}
j-1 \\
r-1
\end{array}\right) \zeta(k-j, \bar{j})+\sum_{j=1}^{k-1}\left(\begin{array}{l}
j-1 \\
s-1
\end{array}\right) \zeta(k-j, \bar{j}) .
$$

Proof.

$\zeta(\bar{r}) \zeta(s)$

$$
\begin{aligned}
= & -\frac{1}{\Gamma(r) \Gamma(s)} \int_{0}^{\infty} \frac{t^{r-1}}{e^{t}+1} d t \int_{0}^{\infty} \frac{u^{s-1}}{e^{u}-1} d u \\
= & -\frac{1}{\Gamma(r) \Gamma(s)} \int_{0}^{\infty} \int_{t}^{\infty} \frac{t^{r-1} u^{s-1}}{\left(e^{t}+1\right)\left(e^{u}-1\right)} d u d t-\frac{1}{\Gamma(r) \Gamma(s)} \int_{0}^{\infty} \int_{u}^{\infty} \frac{t^{r-1} u^{s-1}}{\left(e^{t+1)\left(e^{u}-1\right)}\right.} d t d u \\
= & -\frac{1}{\Gamma(r) \Gamma(s)} \int_{0}^{\infty} \int_{0}^{\infty} \frac{t^{r-1}(t+u)^{s-1}}{\left(e^{t}+1\right)\left(e^{t+u}-1\right)} d s d t-\frac{1}{\Gamma(r) \Gamma(s)} \int_{0}^{\infty} \int_{0}^{\infty} \frac{(t+u)^{r-1} u^{s-1}}{\left(e^{t+u}+1\right)\left(e^{u}-1\right)} d t d u \\
= & \sum_{j=0}^{s-1}\left(\begin{array}{c}
s-1 \\
j
\end{array}\right) \frac{\Gamma(s-j) \Gamma(r+j)}{\Gamma(r) \Gamma(s)} \zeta(\overline{s-j}, \overline{r+j})+\sum_{j=0}^{r-1}\left(\begin{array}{c}
r-1 \\
j
\end{array}\right) \frac{\Gamma(r-j) \Gamma(s+j)}{\Gamma(r) \Gamma(s)} \zeta(\overline{r-j}, s+j) \\
= & \sum_{j=0}^{s-1}\left(\begin{array}{c}
r+j-1 \\
r-1
\end{array}\right) \zeta(\overline{s-j}, \overline{r+j})+\sum_{j=0}^{r-1}\left(\begin{array}{c}
s+j-1 \\
s-1
\end{array}\right) \zeta(\overline{r-j}, s+j) \\
= & \sum_{j=1}^{k-1}\left(\begin{array}{c}
j-1 \\
r-1
\end{array}\right) \zeta(\overline{k-j}, \bar{j})+\sum_{j=1}^{k-1}\left(\begin{array}{c}
j-1 \\
s-1
\end{array}\right) \zeta(\overline{k-j}, j) .
\end{aligned}
$$


The formula (2.10) is proved in the same way.

Before translating these two identities into identities for generating functions, we need to deal with the case $\zeta(\bar{r}) \zeta(s)$ with $s=1$.

Lemma 2.5. If $k \geq 3$,

$$
\begin{aligned}
& \sum_{s=2}^{k-1} \zeta(k-s, s)=\zeta(k) \\
& \sum_{s=2}^{k-1} \zeta(\overline{k-s}, s)=\zeta(\bar{k})+\zeta(1, \overline{k-1})-\zeta(\overline{1}, \overline{k-1}) .
\end{aligned}
$$

Proof. By definition,

$$
\begin{aligned}
\sum_{s=2}^{k-1} \zeta(k-s, s) & =\sum_{1 \leq m<n} \sum_{s=2}^{k-1} \frac{1}{m^{k-s}} \frac{1}{n^{s}} \\
& =\sum_{1 \leq m<n} \frac{1}{m^{k}} \frac{\frac{m^{2}}{n^{2}}\left(1-\left(\frac{m}{n}\right)^{k-2}\right)}{1-\frac{m}{n}} \\
& =\sum_{n=2}^{\infty} \sum_{m=1}^{n-1} \frac{1}{m^{k-2} n(n-m)}-\sum_{n=2}^{\infty} \sum_{m=1}^{n-1} \frac{1}{n^{k-1}(n-m)} .
\end{aligned}
$$

Notice that

$$
\sum_{n=2}^{\infty} \sum_{m=1}^{n-1} \frac{1}{n^{k-1}(n-m)}=\sum_{n=2}^{\infty} \sum_{m=1}^{n-1} \frac{1}{n^{k-1} m}=\zeta(1, k-1) .
$$

On the other hand,

$$
\begin{aligned}
& \sum_{n=2}^{\infty} \sum_{m=1}^{n-1} \frac{1}{m^{k-2} n(n-m)} \\
= & \sum_{m=1}^{\infty} \sum_{n=1}^{\infty} \frac{1}{m^{k-2} n(n+m)} \\
= & \lim _{L \rightarrow \infty} \sum_{m=1}^{\infty} \sum_{n=1}^{L} \frac{1}{m^{k-1}}\left(\frac{1}{n}-\frac{1}{n+m}\right) \\
= & \lim _{L \rightarrow \infty} \sum_{m=1}^{\infty} \frac{1}{m^{k-1}}\left(1+\frac{1}{2}+\ldots+\frac{1}{m}-\frac{1}{L+1}-\ldots-\frac{1}{L+m}\right) \\
= & \sum_{m=1}^{\infty} \sum_{n=1}^{m-1} \frac{1}{m^{k-1}} \frac{1}{n}+\sum_{m=1}^{\infty} \frac{1}{m^{k}}-\lim _{L \rightarrow \infty} \sum_{m=1}^{\infty} \frac{1}{m^{k-1}}\left(\frac{1}{L+1}+\ldots+\frac{1}{L+m}\right) .
\end{aligned}
$$

The first two terms give

$$
\sum_{m=1}^{\infty} \sum_{n=1}^{m-1} \frac{1}{m^{k-1}} \frac{1}{n}+\sum_{m=1}^{\infty} \frac{1}{m^{k}}=\zeta(1, k-1)+\zeta(k) .
$$


For the term that involves $L$, notice that

$$
\begin{aligned}
0 & \leq \sum_{m=1}^{\infty} \frac{1}{m^{k-1}}\left(\frac{1}{L+1}+\ldots+\frac{1}{L+m}\right) \\
& =\sum_{m=1}^{\infty} \frac{1}{m^{k-1}} \sum_{s=1}^{m} \frac{1}{L+s} \\
& =\sum_{s=1}^{\infty} \frac{1}{L+s} \sum_{m=s}^{\infty} \frac{1}{m^{k-1}} \\
& \leq \sum_{s=1}^{\infty} \frac{1}{L+s}\left(\frac{1}{s^{k-1}}+\int_{s}^{\infty} \frac{1}{x^{k-1}} d x\right) \\
& =\sum_{s=1}^{\infty} \frac{1}{L+s} \frac{1}{s^{k-1}}+\frac{1}{k-2} \sum_{s=1}^{\infty} \frac{1}{L+s} \frac{1}{s^{k-2}} \\
& \leq \frac{1}{L} \sum_{s=1}^{\infty} \frac{1}{s^{k-1}}+\frac{1}{k-2} \sum_{s=1}^{\infty} \frac{1}{2 \sqrt{L s}} \frac{1}{s^{k-2}} .
\end{aligned}
$$

Hence, this term goes to 0 uniformly as $L \rightarrow \infty$. This proves that

$$
\sum_{s=2}^{k-1} \zeta(k-s, s)=\zeta(k)
$$

Equation (2.12) is proved in a similarly way. We have

$$
\begin{gathered}
\sum_{s=2}^{k-1} \zeta(\overline{k-s}, s)=\sum_{n=2}^{\infty} \sum_{m=1}^{n-1} \frac{(-1)^{m}}{m^{k-2} n(n-m)}-\sum_{n=2}^{\infty} \sum_{m=1}^{n-1} \frac{(-1)^{m}}{n^{k-1}(n-m)}, \\
\sum_{n=2}^{\infty} \sum_{m=1}^{n-1} \frac{(-1)^{m}}{n^{k-1}(n-m)}=\sum_{n=2}^{\infty} \sum_{m=1}^{n-1} \frac{(-1)^{n-m}}{n^{k-1} m}=\zeta(\overline{1}, \overline{k-1}), \\
\sum_{n=2}^{\infty} \sum_{m=1}^{n-1} \frac{(-1)^{m}}{m^{k-2} n(n-m)}=\sum_{m=2}^{\infty} \sum_{n=1}^{m-1} \frac{(-1)^{m}}{m^{k-1}} \frac{1}{n}+\sum_{m=1}^{\infty} \frac{(-1)^{m}}{m^{k}} \\
-\lim _{L \rightarrow \infty} \sum_{m=1}^{\infty} \frac{(-1)^{m}}{m^{k-1}}\left(\frac{1}{L+1}+\ldots+\frac{1}{L+m}\right) .
\end{gathered}
$$

The first two terms give

$$
\sum_{m=2}^{\infty} \sum_{n=1}^{m-1} \frac{(-1)^{m}}{m^{k-1}} \frac{1}{n}+\sum_{m=1}^{\infty} \frac{(-1)^{m}}{m^{k}}=\zeta(1, \overline{k-1})+\zeta(\bar{k}) .
$$

For the term that involves $L$, since

$$
\left|\sum_{m=1}^{\infty} \frac{(-1)^{m}}{m^{k-1}}\left(\frac{1}{L+1}+\ldots+\frac{1}{L+m}\right)\right| \leq \sum_{m=1}^{\infty} \frac{1}{m^{k-1}}\left(\frac{1}{L+1}+\ldots+\frac{1}{L+m}\right) .
$$

It follows from the previous estimate that this term goes to 0 as $L \rightarrow \infty$. This proves (2.12). 
Corollary 2.6. The relation (2.9) holds formally when $s=1$.

Proof. Put $s=1$ and $r=k-1$ into (2.9) gives

$$
\zeta(\overline{k-1}) \zeta(1)=\zeta(\overline{1}, \overline{k-1})+\sum_{j=1}^{k-1} \zeta(\overline{k-j}, j)
$$

Since formally

$$
\zeta(\overline{k-1}) \zeta(1)=\zeta(\overline{k-1}, 1)+\zeta(1, \overline{k-1})+\zeta(\bar{k})
$$

Eq. (2.13) is equivalent to

$$
\sum_{j=2}^{k-1} \zeta(\overline{k-j}, j)=\zeta(1, \overline{k-1})+\zeta(\bar{k})-\zeta(\overline{1}, \overline{k-1}),
$$

which is equation (2.12). This proves the corollary.

Remark 2.7. Setting $r=1$ in (2.9), we have

$$
\zeta(k-1) \zeta(\overline{1})=\sum_{j=1}^{k-1} \zeta(\overline{k-j}, \bar{j})+\zeta(\overline{1}, k-1) .
$$

Together with the fact that

$$
\zeta(k-1) \zeta(\overline{1})-\zeta(\overline{1}, k-1)=\zeta(\bar{k})+\zeta(k-1, \overline{1}),
$$

we obtain

$$
\sum_{s=2}^{k-1} \zeta(\overline{k-s}, \bar{s})=\zeta(\bar{k})+\zeta(k-1, \overline{1})-\zeta(\overline{k-1}, \overline{1}) .
$$

Similarly, setting $r=1$ in (2.10) gives

$$
\sum_{s=2}^{k-1} \zeta(k-s, \bar{s})=\zeta(\bar{k})+\zeta(\overline{k-1}, \overline{1})+\zeta(\overline{1}, \overline{k-1})-\zeta(k-1, \overline{1})-\zeta(1, \overline{k-1}) .
$$

Eq. (2.12), (2.14) and (2.15) are summation formulas of alternating double Euler sums. They are generalizations of (2.11).

The double shuffle relations in Theorem 2.4 give the following identities of generating functions.

Theorem 2.8.

$$
\begin{aligned}
& F_{1}(x, y)=G_{1}(x, x+y)+G_{3}(y, x+y), \\
& F_{2}(x, y)=G_{2}(x, x+y)+G_{2}(y, x+y), .
\end{aligned}
$$


Proof. Eq. (2.9) gives

$$
\begin{aligned}
& F_{1}(x, y)=\sum_{\substack{r, s \geq 1 \\
r+s=k}} \zeta(\bar{r}) \zeta(s) x^{r-1} y^{s-1} \\
& =\sum_{r=1}^{k-1} \sum_{j=r}^{k-1}\left(\begin{array}{l}
j-1 \\
r-1
\end{array}\right) \zeta(\overline{k-j}, \bar{j}) x^{r-1} y^{k-r-1}+\sum_{s=1}^{k-1} \sum_{j=s}^{k-1}\left(\begin{array}{l}
j-1 \\
s-1
\end{array}\right) \zeta(\overline{k-j}, j) x^{k-s-1} y^{s-1} \\
& =\sum_{j=1}^{k-1}\left[\sum_{r=1}^{j}\left(\begin{array}{l}
j-1 \\
r-1
\end{array}\right) x^{r-1} y^{j-r}\right] \zeta(\overline{k-j}, \bar{j}) y^{k-j-1} \\
& +\sum_{j=1}^{k-1}\left[\sum_{s=1}^{j}\left(\begin{array}{l}
j-1 \\
s-1
\end{array}\right) x^{j-s} y^{s-1}\right] \zeta(\overline{k-j}, j) x^{k-j-1} \\
& =\sum_{j=1}^{k-1} \zeta(\overline{k-j}, \bar{j}) y^{k-j-1}(x+y)^{j-1}+\sum_{j=1}^{k-1} \zeta(\overline{k-j}, j) x^{k-j-1}(x+y)^{j-1} \\
& =G_{3}(y, x+y)+G_{1}(x, x+y)
\end{aligned}
$$

Eq. (2.17) is proved in the same way.

From Theorem 2.2 and Theorem 2.8 one obtains four relations among the functions $F_{1}(x, y), F_{2}(x, y), G_{1}(x, y), G_{2}(x, y)$ and $G_{3}(x, y)$. Our goal is to express $G_{1}$, $G_{2}$ and $G_{3}$ in terms of $F_{1}$ and $F_{2}$.

Theorem 2.9. We have the following relations:

$$
\begin{aligned}
G_{1}(x, y)-G_{1}(-x,-y)= & F_{1}(x, y)-F_{1}(x,-y)-F_{2}(x-y, y)+F_{2}(x-y,-y) \\
& +F_{1}(x, x-y)-F_{1}(-x, x-y) \\
& -T_{2}(x, y)-T_{2}(x, x-y)-T_{1}(x-y,-y) \\
G_{2}(x, y)-G_{2}(-x,-y)= & F_{1}(y, x)-F_{1}(-y, x)-F_{1}(y, x-y)+F_{1}(-y, x-y) \\
& +F_{2}(x, x-y)-F_{2}(-x, x-y) \\
& -T_{2}(x, y)-T_{1}(x, x-y)-T_{2}(x-y,-y) \\
& \\
G_{3}(x, y)-G_{3}(-x,-y)= & F_{2}(x, y)-F_{2}(x,-y)-F_{1}(x-y, y)+F_{1}(x-y,-y) \\
& +F_{1}(x-y, x)-F_{1}(x-y,-x) \\
& -T_{1}(x, y)-T_{2}(x, x-y)-T_{2}(x-y,-y) .
\end{aligned}
$$

Proof. As in [15], we use the relations in Theorem 2.2 and Theorem 2.8 alternately. We have

$$
\begin{aligned}
G_{1}(x, y) & =F_{1}(x, y)-G_{2}(y, x)-T_{2}(x, y) \\
& =F_{1}(x, y)-F_{2}(x-y, y)+G_{2}(x-y, x)-T_{2}(x, y) \\
& =F_{1}(x, y)-F_{2}(x-y, y)+F_{1}(x, x-y)-G_{1}(x, x-y)-T_{2}(x, y)-T_{2}(x, x-y)
\end{aligned}
$$




$$
\begin{aligned}
= & F_{1}(x, y)-F_{2}(x-y, y)+F_{1}(x, x-y)-F_{1}(x,-y)+G_{3}(-y, x-y) \\
& -T_{2}(x, y)-T_{2}(x, x-y) \\
= & F_{1}(x, y)-F_{2}(x-y, y)+F_{1}(x, x-y)-F_{1}(x,-y)+F_{2}(-y, x-y) \\
& -G_{3}(x-y,-y)-T_{2}(x, y)-T_{2}(x, x-y)-T_{1}(-y, x-y) \\
= & F_{1}(x, y)-F_{2}(x-y, y)+F_{1}(x, x-y)-F_{1}(x,-y)+F_{2}(-y, x-y) \\
& -F_{1}(-x, x-y)+G_{1}(-x,-y)-T_{2}(x, y)-T_{2}(x, x-y)-T_{1}(-y, x-y) .
\end{aligned}
$$

This proves (2.18). The other two equations can be proved in the same way.

From Theorem 2.9] we can obtain the main results of this section.

Theorem 2.10. If $k=r+s \geq 3$ is an odd positive integer, then

$$
\begin{aligned}
\zeta(\bar{r}, s)= & -\frac{1}{2} \zeta(\bar{k})+\frac{1+(-1)^{s}}{2} \zeta(\bar{r}) \zeta(s) \\
& +(-1)^{r} \sum_{l=0}^{\frac{k-1}{2}}\left[\left(\begin{array}{c}
k-2 l-1 \\
r-1
\end{array}\right) \zeta(\overline{k-2 l})+\left(\begin{array}{c}
k-2 l-1 \\
s-1
\end{array}\right) \zeta(k-2 l)\right] \zeta(\overline{2 l}), \\
\zeta(r, \bar{s})= & -\frac{1}{2} \zeta(\bar{k})+\frac{1+(-1)^{s}}{2} \zeta(r) \zeta(\bar{s}) \\
& +(-1)^{r} \sum_{l=0}^{\frac{k-1}{2}}\left[\left(\begin{array}{c}
k-2 l-1 \\
r-1
\end{array}\right) \zeta(k-2 l)+\left(\begin{array}{c}
k-2 l-1 \\
s-1
\end{array}\right) \zeta(\overline{k-2 l})\right] \zeta(\overline{2 l}), \\
\zeta(\bar{r}, \bar{s})= & -\frac{1}{2} \zeta(k)+\frac{1+(-1)^{s}}{2} \zeta(\bar{r}) \zeta(\bar{s}) \\
& +(-1)^{r} \sum_{l=0}^{\frac{k-1}{2}}\left[\left(\begin{array}{c}
k-2 l-1 \\
r-1
\end{array}\right) \zeta(\overline{k-2 l})+\left(\begin{array}{c}
k-2 l-1 \\
s-1
\end{array}\right) \zeta(\overline{k-2 l})\right] \zeta(2 l) .
\end{aligned}
$$

Proof. We prove (2.21). The other two formulas can be derived in the same way. We apply the formula (2.18). Since $k$ is odd,

$$
\begin{gathered}
G_{1}(x, y)-G_{1}(-x,-y)=2 \sum_{\substack{r, s \geq 1 \\
r+s=k}} \zeta(\bar{r}, s) x^{r-1} y^{s-1} . \\
F_{1}(x, y)-F_{1}(x,-y)=\sum_{\substack{r, s \geq 1 \\
r+s=k}}\left(1+(-1)^{s}\right) \zeta(\bar{r}) \zeta(s) x^{r-1} y^{s-1} .
\end{gathered}
$$




$$
\begin{aligned}
F_{2}(x-y,-y)-F_{2}(x-y, y) & =-2 \sum_{l=1}^{\frac{k-1}{2}} \zeta(\overline{k-2 l}) \zeta(\overline{2 l})(x-y)^{k-2 l-1} y^{2 l-1} \\
& =-2 \sum_{l=1}^{\frac{k-1}{2}} \zeta(\overline{k-2 l}) \zeta(\overline{2 l}) \sum_{r=1}^{k-1}(-1)^{k-2 l-r}\left(\begin{array}{c}
k-2 l-1 \\
r-1
\end{array}\right) x^{r-1} y^{k-r-1} \\
& =2 \sum_{r=1}^{k-1}\left(\sum_{l=1}^{\frac{k-1}{2}}(-1)^{r}\left(\begin{array}{c}
k-2 l-1 \\
r-1
\end{array}\right) \zeta(\overline{k-2 l}) \zeta(\overline{2 l})\right) x^{r-1} y^{k-r-1} .
\end{aligned}
$$

Similarly,

$$
\begin{aligned}
F_{1}(x, x-y)-F_{1}(-x, x-y) & =2 \sum_{r=1}^{k-1}\left(\sum_{l=1}^{\frac{k-1}{2}}(-1)^{r}\left(\begin{array}{c}
k-2 l-1 \\
k-r-1
\end{array}\right) \zeta(k-2 l) \zeta(\overline{2 l})\right) x^{r-1} y^{k-r-1} . \\
T_{2}(x, x-y) & =\zeta(\bar{k}) \frac{x^{k-1}-(x-y)^{k-1}}{y} \\
& =\zeta(\bar{k}) \sum_{r=1}^{k-1}(-1)^{r}\left(\begin{array}{l}
k-1 \\
r-1
\end{array}\right) x^{r-1} y^{k-r-1} \\
T_{1}(x-y,-y) & =\zeta(k) \frac{(x-y)^{k-1}-(-y)^{k-1}}{x} \\
& =\zeta(k) \sum_{r=1}^{k-1}(-1)^{r}\left(\begin{array}{c}
k-1 \\
k-r-1
\end{array}\right) x^{r-1} y^{k-r-1} .
\end{aligned}
$$

Compare both sides of (2.18) and use the fact that

$$
\zeta(0)=\zeta(\overline{0})=-\frac{1}{2}
$$

give

$$
\begin{aligned}
\zeta(\bar{r}, s)= & -\frac{1}{2} \zeta(\bar{k})+\frac{1+(-1)^{s}}{2} \zeta(\bar{r}) \zeta(s) \\
& +(-1)^{r} \sum_{l=0}^{\frac{k-1}{2}}\left(\begin{array}{c}
k-2 l-1 \\
r-1
\end{array}\right) \zeta(\overline{k-2 l}) \zeta(\overline{2 l})+(-1)^{r} \sum_{l=0}^{\frac{k-1}{2}}\left(\begin{array}{c}
k-2 l-1 \\
s-1
\end{array}\right) \zeta(k-2 l) \zeta(\overline{2 l}),
\end{aligned}
$$

which is the desired result.

From the proof, we see that in deriving the formulas for the alternating Euler sums, we need to use relations that involve $\zeta(\bar{r}, s), \zeta(r, \bar{s})$ and $\zeta(\bar{r}, \bar{s})$ together. Considering anyone of them alone cannot work.

Remark 2.11. In (2.21), $r \geq 1, s \geq 2$ and both sides are well-defined. Similarly, in (2.23), $r \geq 1, s \geq 1$ and both sides are also well-defined. In (2.22), one has to be 
careful when $r=1$. In this case, the equation should read as

$$
\begin{aligned}
\zeta(r, \bar{s})= & -\frac{1}{2} \zeta(\bar{k}) \\
& +(-1)^{r}\left[\begin{array}{l}
\frac{k-3}{2} \\
l=0
\end{array}\left(\begin{array}{c}
k-2 l-1 \\
r-1
\end{array}\right) \zeta(k-2 l) \zeta(\overline{2 l})+\sum_{l=0}^{\frac{k-1}{2}}\left(\begin{array}{c}
k-2 l-1 \\
s-1
\end{array}\right) \zeta(\overline{k-2 l}) \zeta(\overline{2 l})\right] .
\end{aligned}
$$

\section{Hypergeometric Identities}

In this section, we prove a hypergeometric identity that is of interest in its own right. Recall that the generalized hypergeometric function is defined as (see e.g. [1]):

$$
{ }_{p} F_{q}\left[\begin{array}{c}
a_{1}, a_{2}, \ldots, a_{p} \\
b_{1}, b_{2}, \ldots, b_{q}
\end{array} ; x\right]=\sum_{n=0}^{\infty} \frac{\left(a_{1}\right)_{n} \ldots\left(a_{p}\right)_{n}}{\left(b_{1}\right)_{n} \ldots\left(b_{q}\right)_{n}} \frac{x^{n}}{n !} .
$$

Here

$$
(x)_{n}=x(x+1) \ldots(x+n-1)=\frac{\Gamma(x+n)}{\Gamma(x)}
$$

is the Pochhammer symbol, and $b_{i}$ are not negative integers or zero. If $p=q+1$, the series converges absolutely for $|x|<1$. In addition, if

$$
\operatorname{Re}\left(\sum_{i=1}^{q} b_{i}-\sum_{i=1}^{q+1} a_{i}\right)>0,
$$

then the series ${ }_{q+1} F_{q}$ converges absolutely for $|x| \leq 1$. If

$$
0 \geq \operatorname{Re}\left(\sum_{i=1}^{q} b_{i}-\sum_{i=1}^{q+1} a_{i}\right)>-1,
$$

the series ${ }_{q+1} F_{q}$ converges conditionally if $|x|=1$ and $x \neq 1$.

Of special interest are the hypergeometric sums ${ }_{q+1} F_{q}\left[\begin{array}{c}a_{1}, a_{2}, \ldots, a_{q+1} \\ b_{1}, b_{2}, \ldots, b_{q}\end{array} ; \pm 1\right]$. We first quote a few well-known identities.

Lemma 3.1 (Gauss). If $\operatorname{Re}(c-a-b)>0$, then

$$
\sum_{n=0}^{\infty} \frac{(a)_{n}(b)_{n}}{n !(c)_{n}}={ }_{2} F_{1}\left[\begin{array}{c}
a, b \\
c
\end{array} ; 1\right]=\frac{\Gamma(c) \Gamma(c-a-b)}{\Gamma(c-a) \Gamma(c-b)} .
$$

Proof. See [1], page 66.

Lemma 3.2 (Pfaff-Saalschütz).

$$
{ }_{3} F_{2}\left[\begin{array}{c}
a, b,-n \\
c, 1+a+b-c-n
\end{array} ; 1\right]=\frac{(c-a)_{n}(c-b)_{n}}{(c)_{n}(c-a-b)_{n}} .
$$

Proof. See 1], page 69.

As a corollary, we have 


\section{Corollary 3.3.}

$$
\frac{(b)_{n}(c)_{n}}{(1+a-b)_{n}(1+a-c)_{n}}=\sum_{r=0}^{n} \frac{(a+n)_{r}(1+a-b-c)_{r}(-n)_{r}}{r !(1+a-b)_{r}(1+a-c)_{r}} .
$$

Proof. Using the fact that

$$
\begin{aligned}
(x)_{n} & =x(x+1) \ldots(x+n-1) \\
& =(-1)^{n}(1-x-n)(2-x-n) \ldots(-x) \\
& =(-1)^{n}(1-x-n)_{n},
\end{aligned}
$$

we have

$$
\begin{aligned}
\frac{(b)_{n}(c)_{n}}{(1+a-b)_{n}(1+a-c)_{n}} & =\frac{(b)_{n}(1-c-n)_{n}}{(b-a-n)_{n}(1+a-c)_{n}} \\
& ={ }_{3} F_{2}\left[\begin{array}{c}
a+n, 1+a-b-c,-n \\
1+a-b, 1+a-c
\end{array} ; 1\right] \\
& =\sum_{r=0}^{n} \frac{(a+n)_{r}(1+a-b-c)_{r}(-n)_{r}}{r !(1+a-b)_{r}(1+a-c)_{r}} .
\end{aligned}
$$

The following is an identity that can be proved using integral representation of ${ }_{2} F_{1}$.

Lemma 3.4. If $1+a-b$ is not zero or a negative integer, and Re $b<1$,

$$
{ }_{2} F_{1}\left[\begin{array}{c}
a, b \\
1+a-b
\end{array} ;-1\right]=\frac{1}{2} \frac{\Gamma\left(\frac{a}{2}\right) \Gamma(1+a-b)}{\Gamma(a) \Gamma\left(1+\frac{a}{2}-b\right)}
$$

Proof. Using the integral representation (see [1], page 65)

$$
{ }_{2} F_{1}\left[\begin{array}{c}
\alpha, \beta \\
\gamma
\end{array} ; x\right]=\frac{\Gamma(\gamma)}{\Gamma(\beta) \Gamma(\gamma-\beta)} \int_{0}^{1} t^{\beta-1}(1-t)^{\gamma-\beta-1}(1-x t)^{-\alpha} d t,
$$

we find that if $\operatorname{Re} a>0$ and $\operatorname{Re} b<1$,

$$
\begin{aligned}
{ }_{2} F_{1}\left[\begin{array}{c}
a, b \\
1+a-b ;-1]
\end{array}\right. & =\frac{\Gamma(1+a-b)}{\Gamma(a) \Gamma(1-b)} \int_{0}^{1} t^{a-1}(1-t)^{-b}(1+t)^{-b} d t \\
& =\frac{1}{2} \frac{\Gamma(1+a-b)}{\Gamma(a) \Gamma(1-b)} \int_{0}^{1} t^{\frac{a}{2}-1}(1-t)^{-b} d t \\
& =\frac{1}{2} \frac{\Gamma(1+a-b)}{\Gamma(a) \Gamma(1-b)} \frac{\Gamma\left(\frac{a}{2}\right) \Gamma(1-b)}{\Gamma\left(1+\frac{a}{2}-b\right)} \\
& =\frac{1}{2} \frac{\Gamma\left(\frac{a}{2}\right) \Gamma(1+a-b)}{\Gamma(a) \Gamma\left(1+\frac{a}{2}-b\right)} .
\end{aligned}
$$

The results follows from analytic continuation.

Now we prove a special case of our main result. 
Theorem 3.5. If none of $a / 2,1+a-b$ and $1+a-c$ is zero or a negative integer, and $\operatorname{Re}(2+a-2 b-2 c)>0$, then

$$
{ }_{4} F_{3}\left[\begin{array}{c}
a, 1+a / 2, b, c \\
a / 2,1+a-b, 1+a-c
\end{array} ;-1\right]=\frac{\Gamma(1+a-b) \Gamma(1+a-c)}{\Gamma(1+a) \Gamma(1+a-b-c)} .
$$

Proof. This formula can be obtained as a limiting case of Dougall's formula (see for example, [1] ) for a finite ${ }_{7} F_{6}$. Here we give an independent proof that do not use identities for ${ }_{q+1} F_{q}$ with $q \geq 3$.

$$
{ }_{4} F_{3}\left[\begin{array}{c}
a, 1+a / 2, b, c \\
a / 2,1+a-b, 1+a-c
\end{array} ;-1\right]=\sum_{n=0}^{\infty}(-1)^{n} \frac{(a)_{n}(c)_{n}}{n !(1+a-c)_{n}} \frac{(b)_{n}(1+a / 2)_{n}}{(1+a-b)_{n}(a / 2)_{n}}
$$

Using Corollary 3.3 with $c=1+a / 2$, we have

$$
\begin{aligned}
& { }_{4} F_{3}\left[\begin{array}{c}
a, 1+a / 2, b, c \\
a / 2,1+a-b, 1+a-c
\end{array} ;-1\right] \\
= & \sum_{n=0}^{\infty}(-1)^{n} \frac{(a)_{n}(c)_{n}}{n !(1+a-c)_{n}} \sum_{r=0}^{\infty} \frac{(a+n)_{r}(a / 2-b)_{r}(-n)_{r}}{r !(1+a-b)_{r}(a / 2)_{r}} \\
= & \sum_{r=0}^{\infty} \frac{(a / 2-b)_{r}}{r !(1+a-b)_{r}(a / 2)_{r}} \sum_{n=r}^{\infty}(-1)^{n-r} \frac{(a)_{n+r}(c)_{n}}{(n-r) !(1+a-c)_{n}} \\
= & \sum_{r=0}^{\infty} \frac{(a / 2-b)_{r}}{r !(1+a-b)_{r}(a / 2)_{r}} \sum_{n=0}^{\infty}(-1)^{n} \frac{(a)_{n+2 r}(c)_{n+r}}{n !(1+a-c)_{n+r}} \\
= & \sum_{r=0}^{\infty} \frac{(a / 2-b)_{r}(a)_{2 r}(c)_{r}}{r !(a / 2)_{r}(1+a-b)_{r}(1+a-c)_{r}} \sum_{n=0}^{\infty}(-1)^{n} \frac{(a+2 r)_{n}(c+r)_{n}}{n !(1+a-c+r)_{n}} \\
= & \sum_{r=0}^{\infty} \frac{(a / 2-b)_{r}(a)_{2 r}(c)_{r}}{r !(a / 2)_{r}(1+a-b)_{r}(1+a-c)_{r}}{ }_{2} F_{1}\left[\begin{array}{c}
a+2 r, c+r \\
1+a-c+r
\end{array} ;-1\right]
\end{aligned}
$$

Using Lemma 3.4, we find that

$$
\begin{aligned}
& { }_{4} F_{3}\left[\begin{array}{c}
a, 1+a / 2, b, c \\
a / 2,1+a-b, 1+a-c
\end{array} ;-1\right] \\
= & \frac{1}{2} \sum_{r=0}^{\infty} \frac{(a / 2-b)_{r}(a)_{2 r}(c)_{r}}{r !(a / 2)_{r}(1+a-b)_{r}(1+a-c)_{r}} \frac{\Gamma\left(\frac{a}{2}+r\right) \Gamma(1+a-c+r)}{\Gamma(a+2 r) \Gamma\left(1+\frac{a}{2}-c\right)} \\
= & \frac{1}{2} \frac{\Gamma\left(\frac{a}{2}\right) \Gamma(1+a-c)}{\Gamma(a) \Gamma\left(1+\frac{a}{2}-c\right)} \sum_{r=0}^{\infty} \frac{(a / 2-b)_{r}(c)_{r}}{r !(1+a-b)_{r}} \\
= & \frac{\Gamma\left(1+\frac{a}{2}\right) \Gamma(1+a-c)}{\Gamma(1+a) \Gamma\left(1+\frac{a}{2}-c\right)}{ }_{2} F_{1}\left[\begin{array}{c}
a / 2-b, c \\
1+a-b
\end{array} ; 1\right]
\end{aligned}
$$


Lemma 3.1 then gives

$$
\begin{aligned}
{ }_{4} F_{3}\left[\begin{array}{c}
a, 1+a / 2, b, c \\
a / 2,1+a-b, 1+a-c
\end{array} ;-1\right] & =\frac{\Gamma\left(1+\frac{a}{2}\right) \Gamma(1+a-c)}{\Gamma(1+a) \Gamma\left(1+\frac{a}{2}-c\right)} \frac{\Gamma(1+a-b) \Gamma\left(1+\frac{a}{2}-c\right)}{\Gamma\left(1+\frac{a}{2}\right) \Gamma(1+a-b-c)} \\
& =\frac{\Gamma(1+a-b) \Gamma(1+a-c)}{\Gamma(1+a) \Gamma(1+a-b-c)}
\end{aligned}
$$

which proves the theorem.

Finally, we prove the main result of this section.

Theorem 3.6. Let $s \geq 0$. We have the formula

$$
\begin{aligned}
& 2 s+4 F_{2 s+3}\left[\begin{array}{c}
a, 1+a / 2, b_{1}, c_{1}, \ldots, b_{s+1}, c_{s+1} \\
a / 2,1+a-b_{1}, 1+a-c_{1}, \ldots, 1+a-b_{s+1}, 1+a-c_{s+1}
\end{array} ;-1\right] \\
= & \frac{\Gamma\left(1+a-b_{s+1}\right) \Gamma\left(1+a-c_{s+1}\right)}{\Gamma(1+a) \Gamma\left(1+a-b_{s+1}-c_{s+1}\right)} \sum_{k_{1}, k_{2}, \ldots, k_{s} \geq 0} \\
& \times \prod_{j=1}^{s} \frac{\left(1+a-b_{j}-c_{j}\right)_{k_{j}}\left(b_{j+1}\right)_{k_{1}+\ldots+k_{j}}\left(c_{j+1}\right)_{k_{1}+\ldots+k_{j}}}{k_{j} !\left(1+a-b_{j}\right)_{k_{1}+\ldots+k_{j}}\left(1+a-c_{j}\right)_{k_{1}+\ldots+k_{j}}},
\end{aligned}
$$

whenever the left hand side is convergent.

Proof. In [11, this formula is obtained as the limiting case of another hypergeometric identity, which is the $q \rightarrow 1$ limit of a basic hypergeometric identity proved by Andrews 2. Here we give a direct proof using induction on $s$. The case $s=0$ was proved in Theorem 3.5. If $s \geq 1$, using Corollary 3.3. we have

$$
\begin{aligned}
& { }_{2 s+4} F_{2 s+3}\left[\begin{array}{c}
a, 1+a / 2, b_{1}, c_{1}, \ldots, b_{s+1}, c_{s+1} \\
a / 2,1+a-b_{1}, 1+a-c_{1}, \ldots, 1+a-b_{s+1}, 1+a-s_{s+1}
\end{array}\right] \\
= & \sum_{n=0}^{\infty}(-1)^{n} \frac{(a)_{n}(1+a / 2)_{n}\left(b_{1}\right)_{n}\left(c_{1}\right)_{n} \ldots\left(b_{s+1}\right)_{n}\left(c_{s+1}\right)_{n}}{n !(a / 2)_{n}\left(1+a-b_{1}\right)_{n}\left(1+a-c_{1}\right)_{n} \ldots\left(1+a-b_{s+1}\right)_{n}\left(1+a-c_{s+1}\right)_{n}} \\
= & \sum_{n=0}^{\infty}(-1)^{n} \frac{(a)_{n}(1+a / 2)_{n}\left(b_{2}\right)_{n}\left(c_{2}\right)_{n} \ldots\left(b_{s+1}\right)_{n}\left(c_{s+1}\right)_{n}}{n !(a / 2)_{n}\left(1+a-b_{2}\right)_{n}\left(1+a-c_{2}\right)_{n} \ldots\left(1+a-b_{s+1}\right)_{n}\left(1+a-c_{s+1}\right)_{n}} \\
& \times \sum_{r=0}^{n} \frac{(a+n)_{r}\left(1+a-b_{1}-c_{1}\right)_{r}(-n)_{r}}{r !\left(1+a-b_{1}\right)_{r}\left(1+a-c_{1}\right)_{r}} \\
= & \sum_{r=0}^{\infty} \sum_{n=r}^{\infty} \frac{\left(1+a-b_{1}-c_{1}\right)_{r}}{r !\left(1+a-b_{1}\right)_{r}\left(1+a-c_{1}\right)_{r}} \\
& \times(-1)^{n-r} \frac{(a)_{n+r}(1+a / 2)_{n}\left(b_{2}\right)_{n}\left(c_{2}\right)_{n} \ldots\left(b_{s+1}\right)_{n}\left(c_{s+1}\right)_{n}}{(n-r) !(a / 2)_{n}\left(1+a-b_{2}\right)_{n}\left(1+a-c_{2}\right)_{n} \ldots\left(1+a-b_{s+1}\right)_{n}\left(1+a-c_{s+1}\right)_{n}}
\end{aligned}
$$




$$
\begin{aligned}
= & \sum_{r=0}^{\infty} \sum_{n=0}^{\infty} \frac{\left(1+a-b_{1}-c_{1}\right)_{r}}{r !\left(1+a-b_{1}\right)_{r}\left(1+a-c_{1}\right)_{r}} \\
& \times(-1)^{n} \frac{(a)_{n+2 r}(1+a / 2)_{n+r}\left(b_{2}\right)_{n+r}\left(c_{2}\right)_{n+r} \ldots\left(b_{s+1}\right)_{n+r}\left(c_{s+1}\right)_{n+r}}{n !(a / 2)_{n+r}\left(1+a-b_{2}\right)_{n+r}\left(1+a-c_{2}\right)_{n+r} \ldots\left(1+a-b_{s+1}\right)_{n+r}\left(1+a-c_{s+1}\right)_{n+r}} \\
= & \sum_{r=0}^{\infty} \frac{\left(1+a-b_{1}-c_{1}\right)_{r}}{r !\left(1+a-b_{1}\right)_{r}\left(1+a-c_{1}\right)_{r}} \frac{(a)_{2 r}(1+a / 2)_{r} \prod_{j=2}^{s+1}\left(b_{j}\right)_{r}\left(c_{j}\right)_{r}}{(a / 2)_{r} \prod_{j=2}^{s+1}\left(1+a-b_{j}\right)_{r}\left(1+a-c_{j}\right)_{r}} \\
& \times \sum_{n=0}^{\infty}(-1)^{n} \frac{(a+2 r)_{n}(1+a / 2+r)_{n} \prod_{j=2}^{s+1}\left(b_{j}+r\right)_{n}\left(c_{j}+r\right)_{n}}{n !(a / 2+r)_{n} \prod_{j=2}^{s+1}\left(1+a-b_{j}+r\right)_{n}\left(1+a-c_{j}+r\right)_{n}} .
\end{aligned}
$$

By induction hypothesis,

$$
\begin{aligned}
& \sum_{n=0}^{\infty}(-1)^{n} \frac{(a+2 r)_{n}(1+a / 2+r)_{n} \prod_{j=2}^{s+1}\left(b_{j}+r\right)_{n}\left(c_{j}+r\right)_{n}}{n !(a / 2+r)_{n} \prod_{j=2}^{s+1}\left(1+a-b_{j}+r\right)_{n}\left(1+a-c_{j}+r\right)_{n}} \\
= & { }_{2 s+2} F_{2 s+1}\left[\begin{array}{c}
a+2 r, 1+a / 2+r, b_{2}+r, c_{2}+r, \ldots, b_{s+1}+r, c_{s+1}+r \\
a / 2+r, 1+a-b_{2}+r, 1+a-c_{2}+r, \ldots, 1+a-b_{s+1}+r, 1+a-c_{s+1}+r
\end{array}\right] \\
= & \frac{\Gamma\left(1+a-b_{s+1}+r\right) \Gamma\left(1+a-c_{s+1}+r\right)}{\Gamma(1+a+2 r) \Gamma\left(1+a-b_{s+1}-c_{s+1}\right)} \sum_{k_{2}, k_{3}, \ldots, k_{s} \geq 0} \\
& \times \prod_{j=2}^{s} \frac{\left(1+a-b_{j}-c_{j}\right)_{k_{j}}\left(b_{j+1}+r\right)_{k_{2}+\ldots+k_{j}}\left(c_{j+1}+r\right)_{k_{2}+\ldots+k_{j}}}{k_{j} !\left(1+a-b_{j}+r\right)_{k_{2}+\ldots+k_{j}}\left(1+a-c_{j}+r\right)_{k_{2}+\ldots+k_{j}}} .
\end{aligned}
$$

Hence,

$$
\begin{aligned}
& 2 s+4 F_{2 s+3}\left[\begin{array}{c}
a, 1+a / 2, b_{1}, c_{1}, \ldots, b_{s+1}, c_{s+1} \\
a / 2,1+a-b_{1}, 1+a-c_{1}, \ldots, 1+a-b_{s+1}, 1+a-c_{s+1}
\end{array} ;-1\right] \\
= & \sum_{r=0}^{\infty} \frac{\left(1+a-b_{1}-c_{1}\right)_{r}}{r !\left(1+a-b_{1}\right)_{r}\left(1+a-c_{1}\right)_{r}} \frac{(a)_{2 r}(1+a / 2)_{r} \prod_{j=2}^{s+1}\left(b_{j}\right)_{r}\left(c_{j}\right)_{r}}{(a / 2)_{r} \prod_{j=2}^{s+1}\left(1+a-b_{j}\right)_{r}\left(1+a-c_{j}\right)_{r}} \\
& \times \frac{\Gamma\left(1+a-b_{s+1}+r\right) \Gamma\left(1+a-c_{s+1}+r\right)}{\Gamma(1+a+2 r) \Gamma\left(1+a-b_{s+1}-c_{s+1}\right)} \sum_{k_{2}, k_{3}, \ldots, k_{s} \geq 0} \\
& \times \prod_{j=2}^{s} \frac{\left(1+a-b_{j}-c_{j}\right)_{k_{j}}\left(b_{j+1}+r\right)_{k_{2}+\ldots+k_{j}}\left(c_{j+1}+r\right)_{k_{2}+\ldots+k_{j}}}{k_{j} !\left(1+a-b_{j}+r\right)_{k_{2}+\ldots+k_{j}}\left(1+a-c_{j}+r\right)_{k_{2}+\ldots+k_{j}}}
\end{aligned}
$$




$$
\begin{aligned}
= & \frac{\Gamma\left(1+a-b_{s+1}\right) \Gamma\left(1+a-c_{s+1}\right)}{\Gamma(1+a) \Gamma\left(1+a-b_{s+1}-c_{s+1}\right)} \sum_{r, k_{2}, k_{3}, \ldots, k_{s} \geq 0} \frac{\left(1+a-b_{1}-c_{1}\right)_{r}}{r !\left(1+a-b_{1}\right)_{r}\left(1+a-c_{1}\right)_{r}} \\
& \times \frac{a}{a+2 r} \frac{(1+a / 2)_{r}\left(b_{2}\right)_{r}\left(c_{2}\right)_{r}}{(a / 2)_{r}} \sum_{k_{2}, k_{3}, \ldots, k_{s} \geq 0} \\
& \times \prod_{j=2}^{s} \frac{\left(1+a-b_{j}-c_{j}\right)_{k_{j}}\left(b_{j+1}\right)_{r+k_{2}+\ldots+k_{j}}\left(c_{j+1}\right)_{r+k_{2}+\ldots+k_{j}}}{k_{j} !\left(1+a-b_{j}\right)_{r+k_{2}+\ldots+k_{j}}\left(1+a-c_{j}\right)_{r+k_{2}+\ldots+k_{j}}}
\end{aligned}
$$

But

$$
\frac{a}{a+2 r} \frac{(1+a / 2)_{r}}{(a / 2)_{r}}=\frac{a}{a+2 r} \frac{a / 2+r}{a / 2}=1 .
$$

Setting $r=k_{1}$ complete the induction and prove the theorem.

\section{Yet Another Alternative Proof of Zagier's Formula}

In this section, we give another simpler proof of Zagier's formula:

\section{Theorem 4.1.}

$$
\begin{gathered}
H(a, b)=2 \sum_{r=1}^{K}(-1)^{r}\left\{\left(\begin{array}{c}
2 r \\
2 a+2
\end{array}\right) \zeta(2 r+1)+\left(\begin{array}{c}
2 r \\
2 b+1
\end{array}\right) \zeta \overline{(2 r+1)}\right\} H(K-r), \\
H^{\star}(a, b)=-2 \sum_{r=1}^{K}\left\{\left[\left(\begin{array}{c}
2 r \\
2 a
\end{array}\right)-\delta_{r, a}\right] \zeta(2 r+1)+\left(\begin{array}{c}
2 r \\
2 b+1
\end{array}\right) \zeta(\overline{2 r+1})\right\} H^{\star}(K-r),
\end{gathered}
$$

where $K=a+b+1$.

Define the generating functions

$$
\begin{aligned}
G(x) & =\sum_{a=0}^{\infty}(-1)^{a} H(a) x^{2 a}, \\
G^{\star}(x) & =\sum_{a=0}^{\infty} H^{\star}(a) x^{2 a}, \\
F(x, y) & =\sum_{a=0}^{\infty} \sum_{b=0}^{\infty}(-1)^{a+b+1} H(a, b) x^{2 a+2} y^{2 b}, \\
F^{\star}(x, y) & =\sum_{a=0}^{\infty} \sum_{b=0}^{\infty} H^{*}(a, b) x^{2 a} y^{2 b+2} .
\end{aligned}
$$

Here we use the convention that $H(0)=H^{\star}(0)=1$. Notice that our definition is slightly different from those defined in [15] and [9]. 
It is well-known that

$$
\begin{array}{r}
G(x)=\prod_{n=1}^{\infty}\left(1-\frac{x^{2}}{n^{2}}\right)=\frac{\sin \pi x}{\pi x}, \\
G^{\star}(x)=\frac{1}{\prod_{n=1}^{\infty}\left(1-\frac{x^{2}}{n^{2}}\right)}=\frac{\pi x}{\sin \pi x} .
\end{array}
$$

Eq. (4.3) gives immediately

$$
H(a)=\frac{\pi^{2 a}}{(2 a+1) !} .
$$

Using the formula

$$
\begin{aligned}
\frac{\pi x}{\sin \pi x} & =1-2 \sum_{j=1}^{\infty}(-1)^{j} \frac{x^{2}}{j^{2}-x^{2}} \\
& =1-2 \sum_{a=1}^{\infty} \zeta(\overline{2 a}) x^{2 a},
\end{aligned}
$$

we obtain from Eq. (4.4) that for $a \geq 1$,

$$
H^{\star}(a)=-2 \zeta(\overline{2 a}) .
$$

As in [15], we find that for the generating functions $F(x, y)$ and $F^{\star}(x, y)$,

$$
\begin{aligned}
F(x, y) & =-x^{2} \sum_{m=1}^{\infty} \prod_{j=1}^{m-1}\left(1-\frac{x^{2}}{j^{2}}\right) \frac{1}{m^{3}} \prod_{k=m+1}^{\infty}\left(1-\frac{y^{2}}{k^{2}}\right) \\
& =\left.\frac{\sin \pi y}{\pi y} \frac{d}{d z}\right|_{z=0} \sum_{m=1}^{\infty} \frac{(-x)_{m}(x)_{m}}{(1-y)_{m}(1+y)_{m}} z(z+1) \ldots(z+m-1) \frac{1}{m !} \\
& =\left.\frac{\sin \pi y}{\pi y} \frac{d}{d z}\right|_{z=0}{ }_{3} F_{2}\left[\begin{array}{c}
x,-x, z \\
1+y, 1-y
\end{array} ;\right], \\
F^{\star}(x, y) & =y^{2} \sum_{m=1}^{\infty} \prod_{j=1}^{m}\left(1-\frac{x^{2}}{j^{2}}\right)^{-1} \frac{1}{m^{3}} \prod_{k=m}^{\infty}\left(1-\frac{y^{2}}{k^{2}}\right)^{-1} \\
& =-\left.\frac{\pi y}{\sin \pi y} \frac{d}{d z}\right|_{z=0} \sum_{m=1}^{\infty} \frac{(-y)_{m}(y)_{m}}{(1-x)_{m}(1+x)_{m}} z(z+1) \ldots(z+m-1) \frac{1}{m !} \\
& =-\left.\frac{\pi y}{\sin \pi y} \frac{d}{d z}\right|_{z=0}{ }_{3} F_{2}\left[\begin{array}{c}
y,-y, z \\
1+x, 1-x
\end{array} ; 1\right] .
\end{aligned}
$$

Comparing (4.6) and (4.7) give

\section{Lemma 4.2.}

$$
F(x, y)=-\frac{\sin \pi y}{\pi y} \frac{\sin \pi x}{\pi x} F^{\star}(y, x) .
$$

To prove Theorem 4.1, we first give a slightly simpler proof of the following theorem established in [9] and [10]. 
Theorem 4.3. For any $a, b \geq 0$,

$$
H^{\star}(a, b)=-4 \zeta(2 a+1, \overline{2 b+2})-2 \zeta(\overline{2 a+2 b+3}) .
$$

Proof. By Theorem 3.6, we have

$$
\begin{aligned}
& { }_{8} F_{7}\left[\begin{array}{c}
a, 1+a / 2,1+x, 1-x,-\alpha+x,-\alpha-x, y,-y \\
a / 2, a-x, a+x, 1+a+\alpha-x, 1+a+\alpha+x, 1+a-y, 1+a+y
\end{array} ;-1\right] \\
= & \frac{\Gamma(1+a+y) \Gamma(1+a-y)}{\Gamma(1+a)^{2}} \\
& \times \sum_{k_{1}, k_{2} \geq 0} \frac{(a-1)_{k_{1}}(-\alpha+x)_{k_{1}}(-\alpha-x)_{k_{1}}(1+a+2 \alpha)_{k_{2}}(y)_{k_{1}+k_{2}}(-y)_{k_{1}+k_{2}}}{k_{1} ! k_{2} !(a-x)_{k_{1}}(a+x)_{k_{1}}(1+a+\alpha-x)_{k_{1}+k_{2}}(1+a+\alpha+x)_{k_{1}+k_{2}}} .
\end{aligned}
$$

Taking the $a \rightarrow 0$ limit, we find that

$$
\begin{aligned}
& 1+2 \sum_{k=1}^{\infty} \frac{(1+x)_{k}(1-x)_{k}(-\alpha+x)_{k}(-\alpha-x)_{k}(y)_{k}(-y)_{k}}{(-x)_{k}(x)_{k}(1+\alpha-x)_{k}(1+\alpha+x)_{k}(1-y)_{k}(1+y)_{k}}(-1)^{k} \\
= & \Gamma(1+y) \Gamma(1-y)\left\{\sum_{k=0}^{\infty} \frac{(1+2 \alpha)_{k}(y)_{k}(-y)_{k}}{k !(1+\alpha-x)_{k}(1+\alpha+x)_{k}}-\sum_{k=0}^{\infty} \frac{(1+2 \alpha)_{k}(y)_{k+1}(-y)_{k+1}}{k !(1+\alpha-x)_{k+1}(1+\alpha+x)_{k+1}}\right\} \\
= & \frac{\pi y}{\sin \pi y}\left\{1+\sum_{k=1}^{\infty} \frac{(y)_{k}(-y)_{k}}{(1+\alpha-x)_{k}(1+\alpha+x)_{k}}\left[\frac{(1+2 \alpha)_{k}}{k !}-\frac{(1+2 \alpha)_{k-1}}{(k-1) !}\right]\right\} .
\end{aligned}
$$

We then take the derivative of $\alpha$ at $\alpha=0$. The left-hand side gives

$$
2 y^{2} \sum_{k=1}^{\infty} \frac{(-1)^{k}}{k^{2}-y^{2}}\left(4 \sum_{j=1}^{k-1} \frac{j}{j^{2}-x^{2}}+\frac{2 k}{k^{2}-x^{2}}\right),
$$

and the right hand side gives

$$
\begin{aligned}
2 \times \frac{\pi y}{\sin \pi y} \sum_{k=0}^{\infty} \frac{(y)_{k}(-y)_{k}}{(1-x)_{k}(1+x)_{k}} \frac{1}{k} & =2 \times\left.\frac{\pi y}{\sin \pi y} \frac{d}{d z}\right|_{z=0}{ }_{3} F_{2}\left[\begin{array}{c}
y,-y, z \\
1+x, 1-x
\end{array} ; 1\right] \\
& =-2 F^{\star}(x, y) .
\end{aligned}
$$

In other words,

$$
\begin{aligned}
F^{\star}(x, y) & =-y^{2} \sum_{k=1}^{\infty} \frac{(-1)^{k}}{k^{2}-y^{2}}\left(4 \sum_{j=1}^{k-1} \frac{j}{j^{2}-x^{2}}+\frac{2 k}{k^{2}-x^{2}}\right) \\
& =-4 \sum_{a=0}^{\infty} \sum_{b=0}^{\infty} \sum_{k=1}^{\infty} \sum_{j=1}^{k-1} \frac{(-1)^{k}}{k^{2 b+2}} \frac{1}{j^{2 a+1}} x^{2 a} y^{2 b+2}-2 \sum_{a=0}^{\infty} \sum_{b=0}^{\infty} \sum_{k=1}^{\infty} \frac{(-1)^{k}}{k^{2 a+2 b+3}} x^{2 a} y^{2 b+2} \\
& =-\sum_{a=0}^{\infty} \sum_{b=0}^{\infty}(4 \zeta(2 a+1, \overline{2 b+2})+2 \zeta(\overline{2 a+2 b+3})) x^{2 a} y^{2 b+2} .
\end{aligned}
$$

Comparing both sides give

$$
H^{\star}(a, b)=-4 \zeta(2 a+1, \overline{2 b+2})-2 \zeta(\overline{2 a+2 b+3}),
$$

which is the desired result. 
Now we can give a proof to the main theorem Theorem 4.1, which is different from that given in $15,12,9$.

Proof. From Theorem 4.3 and (2.22) in Theorem 2.10, we have

$$
\begin{aligned}
H^{\star}(a, b)= & -4 \zeta(2 a+1) \zeta(\overline{2 b+2}) \\
& +4 \sum_{l=0}^{K}\left[\left(\begin{array}{c}
2 K-2 l \\
2 a
\end{array}\right) \zeta(2 K-2 l+1)+\left(\begin{array}{c}
2 K-2 l \\
2 b+1
\end{array}\right) \zeta(\overline{2 K-2 l+1})\right] \zeta(\overline{2 l}) \\
= & 4 \sum_{r=0}^{K}\left[\left(\begin{array}{l}
2 r \\
2 a
\end{array}\right) \zeta(2 r+1)+\left(\begin{array}{c}
2 r \\
2 b+1
\end{array}\right) \zeta(\overline{2 r+1})\right] \zeta(\overline{2 K-2 r}) \\
& -4 \zeta(2 a+1) \zeta(\overline{2 b+2}) \\
= & -2 \sum_{r=1}^{K}\left\{\left[\left(\begin{array}{c}
2 r \\
2 a
\end{array}\right)-\delta_{r, a}\right] \zeta(2 r+1)+\left(\begin{array}{c}
2 r \\
2 b+1
\end{array}\right) \zeta(\overline{2 r+1})\right\} H^{\star}(K-r) .
\end{aligned}
$$

In the last line, we have used the formula (4.5)).

From this formula for $H^{\star}(a, b)$ and (4.4), we find that

$$
F^{\star}(x, y)=\frac{\pi y}{\sin \pi y} U(x, y)+\frac{\pi x}{\sin \pi x} V(x, y),
$$

where

$$
\begin{aligned}
& U(x, y)=-2 \sum_{a=0}^{\infty} \sum_{\substack{r=a \\
r \geq 1}}^{\infty}\left[\left(\begin{array}{c}
2 r \\
2 a
\end{array}\right)-\delta_{r, a}\right] \zeta(2 r+1) x^{2 a} y^{2 r-2 a}, \\
& V(x, y)=-2 \sum_{b=0}^{\infty} \sum_{r=b+1}^{\infty}\left(\begin{array}{c}
2 r \\
2 b+1
\end{array}\right) \zeta(\overline{2 r+1}) x^{2 r-2 b-2} y^{2 b+2}
\end{aligned}
$$

By Lemma 4.2, we have

$$
F(x, y)=-\frac{\sin \pi y}{\pi y} U(y, x)-\frac{\sin \pi x}{\pi x} V(y, x) .
$$

Now,

$$
\begin{aligned}
U(x, y) & =-2 \sum_{r=1}^{\infty} \sum_{a=0}^{r}\left(\begin{array}{l}
2 r \\
2 a
\end{array}\right) x^{2 a} y^{2 r-2 a} \zeta(2 r+1)+2 \sum_{r=1}^{\infty} \zeta(2 r+1) x^{2 r} \\
& =-\sum_{r=1}^{\infty}\left[(x+y)^{2 r}+(x-y)^{2 r}\right] \zeta(2 r+1)+2 \sum_{r=1}^{\infty} \zeta(2 r+1) x^{2 r} \\
V(x, y) & =-2 \sum_{r=1}^{\infty} \sum_{b=0}^{r-1}\left(\begin{array}{c}
2 r \\
2 b+1
\end{array}\right) x^{2 r-2 b-2} y^{2 b+2} \zeta(\overline{2 r+1}) \\
& =-\frac{y}{x} \sum_{r=1}^{\infty}\left[(x+y)^{2 r}-(x-y)^{2 r}\right] \zeta(\overline{2 r+1}) .
\end{aligned}
$$


Hence,

$$
\begin{aligned}
U(y, x) & =-2 \sum_{r=1}^{\infty} \sum_{a=0}^{r}\left(\begin{array}{c}
2 r \\
2 a
\end{array}\right) \zeta(2 r+1) x^{2 a} y^{2 r-2 a}+2 \sum_{r=1}^{\infty} \zeta(2 r+1) y^{2 r} \\
& =-2 \sum_{a=1}^{\infty} \sum_{r=a}^{\infty}\left(\begin{array}{l}
2 r \\
2 a
\end{array}\right) \zeta(2 r+1) x^{2 a} y^{2 r-2 a} \\
& =-2 \sum_{a=0}^{\infty} \sum_{r=a+1}^{\infty}\left(\begin{array}{c}
2 r \\
2 a+2
\end{array}\right) \zeta(2 r+1) x^{2 a+2} y^{2 r-2 a-2} \\
V(y, x) & =-\frac{x}{y} \sum_{r=1}^{\infty}\left[(x+y)^{2 r}-(x-y)^{2 r}\right] \zeta(\overline{2 r+1}) \\
& =-2 \sum_{b=0}^{\infty} \sum_{r=b+1}^{\infty}\left(\begin{array}{c}
2 r \\
2 b+1
\end{array}\right) \zeta(\overline{2 r+1}) x^{2 r-2 b} y^{2 b} .
\end{aligned}
$$

Comparing both sides of (4.9) and using (4.3) give

$$
H(a, b)=2 \sum_{r=1}^{K}(-1)^{r}\left\{\left(\begin{array}{c}
2 r \\
2 a+2
\end{array}\right) \zeta(2 r+1)+\left(\begin{array}{c}
2 r \\
2 b+1
\end{array}\right) \zeta(\overline{2 r+1})\right\} H(K-r) .
$$

This completes the proof.

An advantage of the proof given here is that it better reflects the symmetries between the formulas for $H(a, b)$ and $H^{\star}(a, b)$.

In the rest of this section, we derive identities for the sums of $H(a, b)$ and $H^{\star}(a, b)$ with fixed $a+b$.

\section{Theorem 4.4.}

$$
\begin{aligned}
\sum_{a+b=K-1} H(a, b) & =\sum_{r=1}^{K}(-1)^{r-1} H(K-r) \zeta(2 r+1), \\
\sum_{a+b=K-1} H^{\star}(a, b) & =\sum_{r=1}^{K} H^{\star}(K-r) \zeta(2 r+1) .
\end{aligned}
$$

Proof. Using (4.6), we have

$$
\begin{aligned}
F(x, x) & =\sum_{a=0}^{\infty} \sum_{b=0}^{\infty}(-1)^{a+b+1} H(a, b) x^{2 a+2 b+2} \\
& =\left.\frac{\sin \pi x}{\pi x} \frac{d}{d z}\right|_{z=0}{ }_{3} F_{2}\left[\begin{array}{c}
x,-x, z \\
1+x, 1-x
\end{array} ; 1\right] .
\end{aligned}
$$

By Theorem 3.4.1 in [1], we have

$$
{ }_{3} F_{2}\left[\begin{array}{c}
x,-x, z \\
1+z+x, 1+z-x
\end{array} ; 1\right]=\frac{\Gamma\left(1+\frac{z}{2}\right)^{2} \Gamma(1+z+x) \Gamma(1+z-x)}{\Gamma(1+z)^{2} \Gamma\left(1+\frac{z}{2}+x\right) \Gamma\left(1+\frac{z}{2}-x\right)} .
$$

Observe that

$$
\left.\frac{d}{d z}\right|_{z=0}{ }_{3} F_{2}\left[\begin{array}{c}
x,-x, z \\
1+x, 1-x
\end{array} ; 1\right]=\left.\frac{d}{d z}\right|_{z=0}{ }_{3} F_{2}\left[\begin{array}{c}
x,-x, z \\
1+z+x, 1+z-x
\end{array} ; 1\right] .
$$


Using

$$
\psi(1+x)=\frac{d}{d x} \log \Gamma(1+x)=\psi(1)+\sum_{n=1}^{\infty}\left(\frac{1}{n}-\frac{1}{n+x}\right),
$$

we find that

$$
\begin{aligned}
\left.\frac{d}{d z}\right|_{z=0}{ }_{3} F_{2}\left[\begin{array}{c}
x,-x, z \\
1+x, 1-x
\end{array} ; 1\right] & =\frac{1}{2}(\psi(1+x)-\psi(1))+\frac{1}{2}(\psi(1-x)-\psi(1)) \\
& =-\sum_{r=1}^{\infty} \zeta(2 r+1) x^{2 r} .
\end{aligned}
$$

It follows from (4.12) and (4.3) that

$$
\sum_{a=0}^{\infty} \sum_{b=0}^{\infty}(-1)^{a+b+1} H(a, b) x^{2 a+2 b+2}=-\sum_{k=0}^{\infty}(-1)^{k} H(k) x^{2 k} \sum_{r=1}^{\infty} \zeta(2 r+1) x^{2 r} .
$$

Comparing both sides give

$$
\sum_{a+b=K-1} H(a, b)=\sum_{r=1}^{K}(-1)^{r-1} H(K-r) \zeta(2 r+1) .
$$

In a similar way, we get

$$
\sum_{a+b=K-1} H^{\star}(a, b)=\sum_{r=1}^{K} H^{\star}(K-r) \zeta(2 r+1) .
$$

\section{Corollary 4.5.}

$$
\zeta(\overline{2 K+1})=-\frac{1}{2 K} \sum_{a+b=K-1}\left(1+\frac{1}{2} \delta_{a, 0}\right) H^{\star}(a, b) .
$$

Proof. Putting $a=0$ in (4.2), we have

$$
H^{\star}(0, K-1)=-2 \sum_{r=1}^{K} \zeta(2 r+1) H^{\star}(K-r)-4 K \zeta(\overline{2 K+1}) .
$$

Eq. (4.11) then gives

$$
-2 K \zeta(\overline{2 K+1})=\sum_{a+b=K-1} H^{\star}(a, b)+\frac{1}{2} H^{\star}(0, K-1),
$$

which gives the desired result.

As mentioned in [15], (4.13) shows that the odd alternating zeta value $\zeta(\overline{2 K+1})$ can be written as a $\mathbb{Q}$-linear combination of $H^{\star}(a, b)$. Eq. (4.5) expresses the even alternating zeta value $\zeta(\overline{2 K})$ as

$$
\zeta(\overline{2 K})=-\frac{1}{2} H^{\star}(K) .
$$

From Theorem 4.3 and Corollary 4.5 , we have 
Corollary 4.6. If $r \geq 0, s \geq 1$, then

$$
\zeta(2 r+1, \overline{2 s})=\frac{1}{4 K} \sum_{a+b=K-1}\left(1+\frac{1}{2} \delta_{a, 0}-K \delta_{a, r}\right) H^{\star}(a, b),
$$

where $r+s=K$.

Proof. From (4.8), we have

$$
\zeta(2 r+1, \overline{2 s})=-\frac{1}{4} H^{\star}(r, s-1)-\frac{1}{2} \zeta(\overline{2 K+1}) .
$$

One then obtains from (4.13) that

$$
\begin{aligned}
\zeta(2 r+1, \overline{2 s}) & =-\frac{1}{4} H^{\star}(r, s-1)+\frac{1}{4 K} \sum_{a+b=K-1}\left(1+\frac{1}{2} \delta_{a, 0}\right) H^{\star}(a, b) \\
& =\frac{1}{4 K} \sum_{a+b=K-1}\left(1+\frac{1}{2} \delta_{a, 0}-K \delta_{a, r}\right) H^{\star}(a, b) .
\end{aligned}
$$

In this corollary, the alternating double zeta value $\zeta(2 r+1, \overline{2 s})$ is explicitly expressed in terms of $H^{\star}(a, b)$. An interesting question is whether it is possible to explicitly express all alternating double zeta values in terms of $H^{\star}(a)$ and $H^{\star}(a, b)$.

\section{REFERENCES}

1. G. E. Andrews, R. Askey and R. Roy, Special functions, Cambridge University Press, 2000.

2. G. E. Andrews, Problems and prospects for basic hypergeometric functions, in Theory and application of special functions, Math. Res. Center, Univ. Wisconsin, Publ. No. 35, Academic Press, New York, 1975, 191- 224.

3. D. Borwein, J. M. Borwein and R. Girgensohn, Explicit evaluation of Euler sums, Proceedings of the Edinburgh Mathematical Society 38 (1995), 277-294.

4. F. Brown, Mixed Tate motives over $\mathbb{Z}$, Ann. Math. 175 (2012), 949-976.

5. P. Cartier, On the double zeta values, IHES/M/11/21, 2011.

6. R. E. Crandall and J. P. Buhler, On the evaluation of Euler sums, Experimental Mathematics 3 (1994), 275-285.

7. P. Flajolet, B. Salvy, Euler sums and contour integral representations, Experiment. Math. 7 (1998), 15-35.

8. H. Gangl, M. Kaneko and D. Zagier, Double zeta values and modular forms, in Automorphic Forms and Zeta Functions, World Sci. Publ., Hackensack, NJ, 2006, 71-106.

9. Kh. Hessami Pilehrood, T. Hessami Pilehrood, An alternative proof of a theorem of Zagier, J. Math. Anal. Appl. 449 (2017), 168-175.

10. Kh. Hessami Pilehrood, T. Hessami Pilehrood, R. Tauraso, New properties of multiple harmonic sums modulo $p$ and p-analogues of Leshchiner's series, Trans. Amer. Math. Soc. 366 (2014), 3131-3159.

11. C. Krattenhaler and T. Rivoal, An identity of Andrews, multiple integrals, and very-wellpoised hypergeometric series, Ramanujan J. 13 (2007), 203-219.

12. Z. H. Li, Another proof of Zagier's evaluation formula of the multiple zeta values $\zeta(2, \ldots, 2,3,2, \ldots, 2)$, Math. Res. Lett. 20 (2013), 947-950.

13. T. Nakamura and K. Tasaka, Remarks on double zeta values of level 2, J. Number Theory 133 (2013), 48-54.

14. Z. Xia, T. Cai, D.M. Bradley, Signed q-analogs of Tornheim's double series, Proc. Amer. Math. Soc. 136 (2008), 2689-2698.

15. D. Zagier, Evaluation of the multiple zeta values $\zeta(2, \ldots, 2,3,2, \ldots, 2)$, Ann. Math. 175 (2012), 977-1000. 
Department of Mathematics, Xiamen University Malaysia, Jalan Sunsuria, Bandar Sunsuria, 43900, Sepang, Selangor, Malaysia.

E-mail address: lpteo@xmu.edu.my 